\title{
Erratum to: Novel polystyrene/antibody nanoparticle-coated capillary for immunoaffinity in-tube solid-phase microextraction
}

\author{
Bei Xu ${ }^{1,2} \cdot$ Shuai Cheng ${ }^{1} \cdot X_{\text {Xianhua Wang }}{ }^{1} \cdot$ Dongmei Wang ${ }^{1} \cdot \operatorname{Liang} \mathrm{Xu}^{1}$
}

Received: 28 April 2015 / Accepted: 28 April 2015 /Published online: 10 May 2015

(C) Springer-Verlag Berlin Heidelberg 2015

Erratum to: Anal Bioanal Chem

DOI 10.1007/s00216-014-8419-y

The authors would like to call the reader's attention to the following:

Unfortunately a wrong project number was printed in the section "Acknowledgments".

The paragraph should correctly read:

\section{Acknowledgments}

This work was financially supported by the National Natural Science Foundation of China (No. 81402889) and Natural Science Foundation of Tianjin (No. 14JCYBJC24300). We would like to thank Mr. Haifeng Zhao (Epsilon Bio. Tech. Co.) for his valuable discussion and suggestion.

The online version of the original article can be found at http://dx.doi.org/ 10.1007/s00216-014-8419-y.

Liang Xu

xuliang@tmu.edu.cn

1 Tianjin Key Laboratory on Technologies Enabling Development of Clinical Therapeutics and Diagnostics, School of Pharmacy, Tianjin Medical University, Tianjin 300070, China

2 School of Public Health, Tianjin Medical University, Tianjin 300070 , China 